\title{
Daily intake of Lactobacillus casei Shirota increases natural killer cell activity in smokers
}

\author{
Marcella Reale ${ }^{1,2}$, Paolo Boscolo ${ }^{3}$, Veronica Bellante ${ }^{1}$, Chiara Tarantelli ${ }^{1}$, Marta Di Nicola ${ }^{3}$, \\ Laura Forcella ${ }^{3}$, Qing $\mathrm{Li}^{4}$, Kanehisa Morimoto ${ }^{5}$ and Raffaella Muraro ${ }^{1,2 *}$ \\ ${ }^{1}$ Department of Oncology and Experimental Medicine, Nuovo Polo Didattico, Edificio C, III livello, Via dei Vestini, 31, \\ University "G. d'Annunzio", 66013 Chieti, Italy \\ ${ }^{2}$ Center of Excellence on Aging, Ce.S.I., "G. d'Annunzio" University Foundation, Chieti, Italy \\ ${ }^{3}$ Department of Biomedical Sciences, University "G. d'Annunzio", Chieti, Italy \\ ${ }^{4}$ Department of Hygiene and Public Health, Nippon Medical School, Bunkyo-ku, Tokyo, Japan \\ ${ }^{5}$ Department of Social and Environmental Medicine, Osaka University Graduate School of Medicine, Osaka, Japan
}

(Submitted 10 March 2011 - Final revision received 15 September 2011 - Accepted 16 September 2011 - First published online 6 December 2011)

\begin{abstract}
Dietary probiotics supplementation exerts beneficial health effects. Since cigarette smoking reduces natural killer (NK) activity, we evaluated the effect of Lactobacillus casei Shirota (LcS) intake on NK cytotoxic activity in male smokers. The double-blind, placebo-controlled, randomised study was conducted on seventy-two healthy Italian blue-collar male smokers randomly divided for daily intake of LcS powder or placebo. Before and after 3 weeks of intake, peripheral blood mononuclear cells were isolated and NK activity and CD16 ${ }^{+}$cells' number were assessed. Daily LcS intake for 3 weeks significantly increased NK activity $(P<0 \cdot 001)$. The increase in NK activity was paralleled by an increase in $\mathrm{CD}^{+} 6^{+}$cells $(P<0 \cdot 001)$. Before intake, NK cytotoxic activity inversely correlated with the number of cigarettes smoked $(R-0.064)$. LcS intake prevented the smoke-dependent expected NK activity reduction. The analysis of the distribution of changes in smoke-adjusted NK activity demonstrated that the positive variations were significantly associated with LcS intake, while the negative variations were associated with placebo intake (median value of distributions of differences, $20 \cdot 98$ lytic unit (LU) $/ 10^{7}$ cells for LcS $v$. $-4.38 \mathrm{LU} / 10^{7}$ cells for placebo, $\left.P=0.039\right)$. In conclusion, 3 weeks of daily LcS intake in Italian male smokers was associated with a higher increase in cytotoxic activity and $\mathrm{CD}_{1} 6^{+}$cells' number in comparison to the placebo intake group.
\end{abstract}

Key words: Probiotics: Lactobacillus casei Shirota: Natural killer cells: Smoking habit

Epidemiological studies indicate that dietary probiotics supplementation may exert a wide range of beneficial health effects on humans: from reducing infections and seasonal illness, to decreasing symptoms' perception or duration ${ }^{(1-3)}$. However, the precise mechanisms influencing probiotichost crosstalk are still largely unclear. Probiotics appear to exert a pleiotropic effect resulting chiefly in a protective role on the intestinal tract where they exert direct antimicrobial effects, by competing with enteric pathogens and indirectly by enhancing the intestinal barrier functions ${ }^{(4)}$. Most interesting of these appears to be the probiotics' capability to modulate host's defences, both local and systemic mucosal immune systems ${ }^{(5)}$. It has been shown that probiotics can shape the mucosal immune system towards a non-inflammatory, tolerogenic pattern by increasing IL-10 levels $^{(6)}$, the induction of $\mathrm{CD}^{+}{ }^{+} \mathrm{Foxp}^{+}$regulatory $\mathrm{T}$ cells and by inhibiting the production of pro-inflammatory cytokines, but also may skew T cells towards T helper 1 polarisation ${ }^{(7-10)}$. The predominant cytokine profile depends on the nature and strength of the stimulus and strain of probiotic bacteria ${ }^{(11-13)}$.

Dietary probiotic supplementation could represent an effective instrument to improve natural killer (NK) activity particularly in the elderly ${ }^{(2,3,14,15)}$. In fact, increased risk of diseases and higher mortality rates correlate with the age-related progressive decline of lymphoid cell activity ${ }^{(16,17)}$. However, senescence does not represent the only factor of immune function impairment, but also particular lifestyles may negatively influence the immune functions ${ }^{(2,18)}$. Reduced NK or lymphokine-activated killer activities were detected in the young with depressive disorders ${ }^{(19)}$, or in subjects after acute stress (i.e. victims of an earthquake, or women after breast biopsy) ${ }^{(20,21)}$ and in individuals with occupational stress, increased job demand (JD), repetitive or day/night work shifts or in conditions of job insecurity ${ }^{(22-24)}$. In contrast,

Abbreviations: CPM, counts per min; DL, decision latitude; E, effector; JD, job demand; JS, job strain; LcS, Lactobacillus casei Shirota; LU, lytic unit; NK, natural killer; PBMC, peripheral blood mononuclear cells; SS, social support; STAI, state-trait-anxiety inventory; T, target. 
higher NK activities were associated with healthy lifestyle and stable mental conditions ${ }^{(25-27)}$. Physical activity was shown to improve NK activity, to increase the number of $\mathrm{CD} 16^{+}$ cells and of anticancer-protein expressing cells as well as to induce a general reduction of anxiety and depression ${ }^{(25,28,29)}$. However, in healthy smokers, NK activity is reduced and inversely correlated with the number of cigarettes smoked $^{(25,30)}$. Recently, additional evidence indicated that cigarette smoking impairs NK cell-dependent tumour immune surveillance ${ }^{(31)}$. Thus, the means of enhancing NK-mediated immunity are most desirable.

In the present study, we sought to evaluate the effect of Lactobacillus casei Shirota (LcS) intake on NK-mediated activity in a population of Italian males with different smoking habits.

\section{Materials and methods}

\section{Subjects}

A total of seventy-two healthy Italian blue-collar male subjects with smoking habits (more than five cigarettes/d for at least 5 years) and age ranging between 40 and 60 years were recruited for the study. Table 1 summarises the main physical parameters of the individuals enrolled in the study as well as the number of cigarettes smoked (cigarettes/d average 19.95, range 5-40) with an average pack/ year consumption of $364 \cdot 1$ (range $91 \cdot 2-730 \cdot 0$ ). Exclusion criteria included any medical condition or the use of medications that could interfere with the probiotic intake in a period of 4 weeks before the start of or during the study. Among the seventy-two subjects enrolled, seven individuals were not included in the study owing to dropout/non-compliance/technical/procedural reasons.

The study was conducted at the University "G. d'Annunzio" of Chieti-Pescara, Italy and performed in accordance with the guidelines laid down in the Declaration of Helsinki and was approved by the Ethics Committee of the University "G. d'Annunzio". Written informed consent was obtained from all individuals enrolled in the study.

\section{Experimental design}

The placebo-controlled, double-blind study was performed by randomly dividing the seventy-two subjects into two groups: a group received LcS powder (from now referred to as 'LcS')

Table 1. Characteristics of subjects enrolled in the randomised study (Mean values and standard deviations)

\begin{tabular}{lrrrrrr}
\hline & \multicolumn{2}{c}{ Placebo group } & & \multicolumn{2}{c}{ LcS group } & \\
\cline { 2 - 3 } Variables & Mean & \multicolumn{1}{c}{ SD } & & Mean & SD & \multirow{2}{*}{$P^{*}$} \\
\hline Age (years) & 49.2 & 6.5 & & 51.4 & 6.2 & 0.150 \\
Height $(\mathrm{cm})$ & 174.5 & 6.0 & & 174.5 & 6.9 & 0.879 \\
Weight $(\mathrm{kg})$ & 79.7 & 10.1 & & 81.4 & 11.5 & 0.535 \\
BMI $\left(\mathrm{kg} / \mathrm{m}^{2}\right)$ & 26.1 & 2.8 & & 26.7 & 3.5 & 0.604 \\
No. of cigarettes/d & 19.2 & 8.9 & & 20.7 & 9.7 & 0.386 \\
\hline
\end{tabular}

LcS, Lactobacillus casei Shirota.

${ }^{\star}$ Mann-Whitney: placebo v. LcS. and a group received a powder without LcS (from now referred to as 'placebo') orally every day for 3 weeks. For allocation of the subjects, a computer-generated list of random numbers was used. Participants were assigned following equal randomisation (1:1) to the LCS or placebo intake group.

The L. casei powder (LCS powder) preparation contained about $1 \times 10^{10}$ lyophilised viable LcS strain per gram per sachet (four sachets/d for 3 weeks). The placebo preparation contained only sterile maize starch powder. LcS and placebocontaining sachets were reconstituted using cold water just before intake. LcS and placebo preparations were provided by Yakult Honsha Company Limited, Tokyo, Japan. All individuals were supplied with the total number of sachets to be used for the 3-week intake. Compliance was based both on self-reporting to the monitoring medical staff and by verification of the number of sachets left during and at the end of the study. Randomisation codes were disclosed after experimental data collection and statistical analyses.

\section{Determination of natural killer activity}

NK activity was measured using peripheral blood mononuclear cells (PBMC) isolated from $8 \mathrm{ml}$ of heparinised blood using BD Vacutainer CPT (Becton Dickinson, Franklin Laker, NJ, USA). PBMC were resuspended in Roswell Park Memorial Institute (RPMI) 1640 with 10\% fetal bovine serum (Sigma-Aldrich Co., St Louis, MO, USA) and used as effector cells. The K562 human erythroleukaemia cell line was used as the target for determining NK cell cytotoxicity ${ }^{(29)}$. K562 cells at $1 \times 10^{7} / \mathrm{ml}$ were labelled with $100 \mu \mathrm{Ci}$ of ${ }^{51} \mathrm{Cr}$ for $60 \mathrm{~min}$ at $37^{\circ} \mathrm{C}\left({ }^{51} \mathrm{Cr}\right.$; Perkin Elmer, Waltham, MA, USA $)$ plated into round-bottomed ninety-six-well plates at $1 \times 10^{4} /$ well, mixed with effector cells at different effector target cell ratios (E:T 40:1, 20:1 and 10:1) and incubated at $37^{\circ} \mathrm{C}$ for $4 \mathrm{~h}$. Supernatants from each well were collected after centrifugation and measured in a gamma counter. All experiments were performed in triplicate. The percentage of specific lysis was determined according to the following formula: specific lysis $=($ mean counts per $\min (\mathrm{cpm})$ experimental release - mean cpm spontaneous release)/(mean cpm maximal release - mean $\mathrm{cpm}$ spontaneous release $) \times 100$. One lytic unit (LU) was defined as the cytotoxic activity giving $33.3 \%$ of maximal release.

\section{Flow cytometry}

The numbers of leucocytes, neutrophils, monocytes, eosinophils and lymphocytes were determined by haemocytometric analysis. $\mathrm{CD} 16^{+}$lymphocytes were determined using phycoerythrin (PE)-conjugated anti-CD16 antibodies (Becton Dickinson, San Jose, CA, USA) by flow cytometry performed using the FACScalibur platform (BD Biosciences, Franklin Lakes, NJ, USA).

\section{Analysis of anxiety, occupational stress and perception of symptoms}

The questionnaires for determining the perception of anxiety, occupational stress and subjective symptoms were 
administered before and after intake. State-trait-anxiety inventory (STAI) was used in Scale I to measure state-anxiety as a temporary and varying condition, and in Scale II to monitor trait-anxiety, as a relatively fixed personality tendency $^{(32,33)}$. Occupational stress was determined by the Karasek test including nine items for decision latitude (DL, skill decision + decision authority), twenty for JD, eight for social support (SS) and nine for job insecurity ${ }^{(34-36)}$. Job strain (JS) was determined by the ratio JD:DL. Perception of subjective symptoms (e.g. headache, nausea, stomach-ache) was determined by the Somatisation scale of Symptom Checklist SCL-90 evaluated on a Likert scale ranging from $0=$ not at all to $5=$ very much.

\section{Statistical analysis}

A total of sixty subjects (thirty for the LcS group and thirty for the placebo group) were required to provide $80 \%$ power to detect, at the 0.05 two-sided level, a difference between the group proportions of NK cell activity of $30 \%$. Assuming a $20 \%$ dropout rate, seventy-two subjects were enrolled and randomised for the two groups of the study.

Quantitative variables were summarised as mean and standard deviation, while the qualitative variables were expressed as frequency and percentage. Statistical significance of differences between groups for qualitative variables was assessed using the $\chi^{2}$ test or Fisher's exact test. Non-parametric Mann-Whitney $U$ test was applied for assessing the comparison of the quantitative variables between the placebo and LcS groups, while the Wilcoxon rank sum test was applied for within-group comparison. Spearman's $\rho$ correlation coefficient was applied to evaluate the correlation between quantitative variables. Linear regression analysis was applied to evaluate the relationship between NK cell activity, expressed as LU and smoking habits, expressed as linear combination $\left(0.5 X_{1}+X_{2}\right)$ of number of cigarettes daily smoked $\left(X_{1}\right)$ and number of smoked cigarettes before blood bleed $\left(X_{2}\right)$. The linear relationship estimated was used to adjust NK cell activity measured before and after the intake. The differences between the adjusted values before and after intake were summarised as median and interquartile range. Data were analysed by SPSS ${ }^{\circledR}$ Advanced Statistical ${ }^{\mathrm{TM}}$ version 13.0, 2004 (SPSS Inc., Chicago, IL, USA).

\section{Results}

\section{Subject characteristics}

The baseline physical characteristics, age, weight and BMI as well as smoking habits, were similar among all seventy-two healthy males enrolled in the randomised, double-blind, placebo-controlled study to evaluate the effect of LcS intake (Table 1). Similarly, leucocyte distribution of all individuals did not change after placebo or LcS intake (Table 2).

\section{Effect of Lactobacillus casei Shirota intake on natural killer cell activity}

NK activity was assessed before and after LcS or placebo intake using PBMC isolated immediately after blood sampling. Table 3 shows NK-mediated cytotoxic activities at different E:T ratios before and after LcS and placebo intake. Different starting NK activity levels were detected in LcS and placebo intake groups, although these differences were not statistically significant. Overall, significant NK activity increases were detected after LcS intake at all E:T ratios (range 16.9-26.0\%) as compared to placebo intake (range 8.4-10.5\%). These observations were confirmed by calculating the LU for each individual and for each group. While the increase in LU for the placebo was not statistically significant, a highly significant increase in LU $(127.0 \%, P<0.001)$ was associated with LcS intake. However, due to the apparently lower NK basal levels in the LcS group, the LcS-dependent increase in NK activity not necessarily represents an improved NK cell activity over the placebo group.

Although LcS and placebo intake did not modify the leucocyte distribution (Table 2), significant increases in $\mathrm{CD}_{16}{ }^{+}$cells' numbers in the $\operatorname{LcS}(50 \%, P<0.001)$ and placebo $(20 \%$, $P=0.007$ ) intake groups were observed (Table 3). The $\mathrm{CD} 6^{+}$cell number increase after LcS intake significantly and directly correlated with NK cytotoxicity $(\rho=0.496$, $P<0 \cdot 01)$ as compared to placebo intake $(\rho=0 \cdot 057$, NS).

Fig. 1 illustrates NK activity relative variations in the placebo and in the LcS intake groups. It is to be noted that minimal changes occurred within the placebo group (median 0.25), while appreciable variations were observed after LcS-intake (median $1 \cdot 26$, LcS $v$. placebo; $P=0 \cdot 059$ ).

Table 2. Leucocyte subpopulations in the placebo and Lactobacillus casei Shirota (LcS) intake groups (Mean values and standard deviations)

\begin{tabular}{|c|c|c|c|c|c|c|c|c|}
\hline \multirow[b]{3}{*}{ Cell types } & \multicolumn{4}{|c|}{ Placebo group } & \multicolumn{4}{|c|}{ LcS group } \\
\hline & \multicolumn{2}{|c|}{$\begin{array}{l}\text { Before intake } \\
\left(n \times 10^{-3} / \mathrm{mm}^{3}\right)\end{array}$} & \multicolumn{2}{|c|}{$\begin{array}{c}\text { After intake } \\
\left(n \times 10^{-3} / \mathrm{mm}^{3}\right)\end{array}$} & \multicolumn{2}{|c|}{$\begin{array}{c}\text { Before intake } \\
\left(n \times 10^{-3} / \mathrm{mm}^{3}\right)\end{array}$} & \multicolumn{2}{|c|}{$\begin{array}{c}\text { After intake } \\
\left(n \times 10^{-3} / \mathrm{mm}^{3}\right)\end{array}$} \\
\hline & Mean & SD & Mean & SD & Mean & SD & Mean & SD \\
\hline Leucocytes & $7 \cdot 0$ & 1.7 & $7 \cdot 0$ & $2 \cdot 0$ & $7 \cdot 1$ & 1.8 & $6 \cdot 9$ & 1.8 \\
\hline Neutrophils & 4.0 & 1.6 & $4 \cdot 1$ & 1.9 & $4 \cdot 4$ & 1.5 & $4 \cdot 2$ & 1.4 \\
\hline Monocytes & 0.4 & 0.2 & 0.4 & 0.1 & 0.5 & $1 \cdot 1$ & 0.4 & 0.1 \\
\hline Eosinophils & 0.2 & 0.2 & 0.2 & 0.2 & 0.2 & 0.1 & 0.2 & 0.1 \\
\hline Lymphocytes & $2 \cdot 3$ & 0.6 & $2 \cdot 2$ & 0.5 & $2 \cdot 0$ & 0.6 & $2 \cdot 0$ & 0.6 \\
\hline
\end{tabular}


Table 3. Natural killer (NK) cytotoxic activity, lytic units (LU) and $\mathrm{CD} 16^{+}$in the Lactobacillus casei Shirota (LCS) and placebo intake groups (Mean values and standard deviations) ${ }^{*}$

\begin{tabular}{|c|c|c|c|c|c|c|c|c|c|c|c|c|}
\hline \multirow[b]{3}{*}{ NK cytotoxicity } & \multicolumn{5}{|c|}{ Placebo group } & & \multicolumn{5}{|c|}{ LcS group } & \multirow[b]{3}{*}{$\Delta \% \ddagger$} \\
\hline & \multicolumn{2}{|c|}{ Before intake } & \multicolumn{2}{|c|}{ After intake } & \multirow[b]{2}{*}{$P \dagger$} & \multirow[b]{2}{*}{$\Delta \% \ddagger$} & \multicolumn{2}{|c|}{ Before intake } & \multicolumn{2}{|c|}{ After intake } & \multirow[b]{2}{*}{$P \dagger$} & \\
\hline & Mean & SD & Mean & SD & & & Mean & SD & Mean & SD & & \\
\hline E:T ratio $40: 1$ & $50 \cdot 8$ & $18 \cdot 2$ & $55 \cdot 3$ & $15 \cdot 1$ & 0.150 & $8 \cdot 8$ & $46 \cdot 8$ & $19 \cdot 0$ & $54 \cdot 7$ & $16 \cdot 1$ & 0.017 & $16 \cdot 9$ \\
\hline $\mathrm{E}: \mathrm{T}$ ratio $20: 1$ & $39 \cdot 2$ & $17 \cdot 4$ & $42 \cdot 5$ & $15 \cdot 3$ & 0.242 & 8.4 & $34 \cdot 1$ & $16 \cdot 1$ & 41.9 & $15 \cdot 5$ & 0.001 & $22 \cdot 9$ \\
\hline $\mathrm{E}: \mathrm{T}$ ratio $10: 1$ & 27.5 & $15 \cdot 2$ & $30 \cdot 4$ & $12 \cdot 8$ & 0.153 & $10 \cdot 5$ & $23 \cdot 4$ & $12 \cdot 3$ & $29 \cdot 6$ & $14 \cdot 3$ & 0.002 & $26 \cdot 5$ \\
\hline LU & $104 \cdot 2$ & $144 \cdot 7$ & $151 \cdot 1$ & 228.6 & 0.140 & $45 \cdot 0$ & $70 \cdot 1$ & 61.3 & $159 \cdot 3$ & $290 \cdot 6$ & $<0.001$ & $127 \cdot 2$ \\
\hline $\mathrm{CD}_{16}^{+}$cell no. & 0.5 & 0.2 & 0.6 & 0.2 & 0.007 & $20 \cdot 0$ & 0.4 & 0.2 & 0.6 & 0.2 & $<0.001$ & $50 \cdot 0$ \\
\hline
\end{tabular}

${ }^{*} \mathrm{NK}$ activity was expressed as mean and standard deviation of \% cytotoxicity at indicated E:T ratios and as LU representing the number of effector cells required to lyse $33 \%$ of target cells.

†Wilcoxon signed ranks test: before intake $v$. after intake.

$\ddagger$ The mean of relative differences of all subjects.

Smoking habits and natural killer cell activity: effect of Lactobacillus casei Shirota or placebo intake

The number of cigarettes daily smoked by all the enrolled subjects was carefully monitored to ensure that this did not change during the 3 -week treatment. The smoking habits were calculated by the linear combination of the number of cigarettes smoked the day before $\left(X_{1}\right)$ and the same day just before blood bleed $\left(X_{2}\right)$, as previously indicated by Morimoto et $a l{ }^{(2)}$. The smoking habit of each subject was calculated on a daily scale only to represent the long-term smoking habits of all individuals, and not to indicate that the negative effects of smoke would be over in $1 \mathrm{~d}$. The effect of cigarette smoking on NK activity (expressed as LU) in all subjects is represented in Fig. 2(a). It appears evident that there is an inverse correlation between smoking habits and NK lytic activities in all individuals before LcS or placebo intake. In fact, heavier smokers displayed lower NK activity, while higher NK activities were associated with light smoking habits.

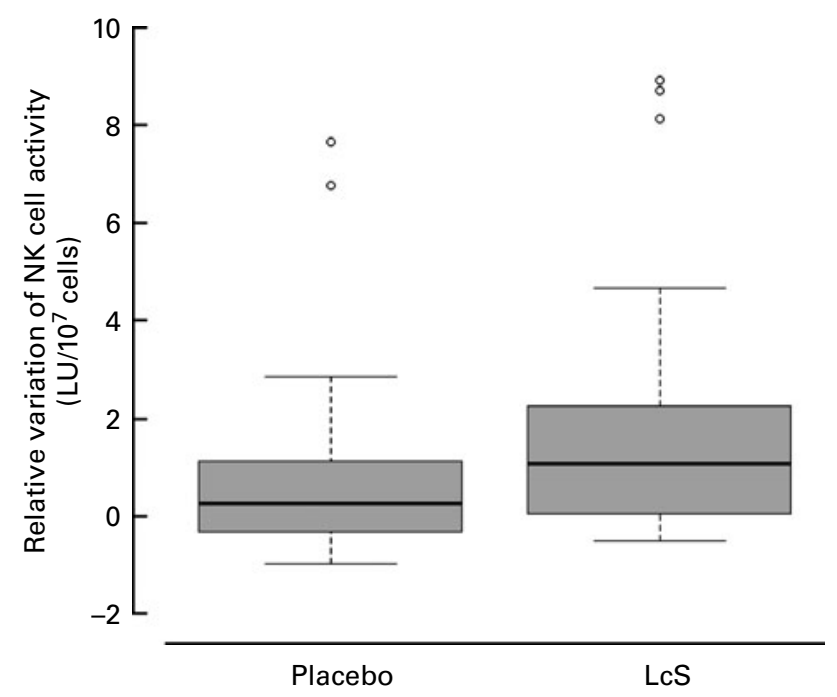

Fig. 1. Relative variation of natural killer (NK) cell activity in the placebo and Lactobacillus casei Shirota (LCS) intake groups. Box-whisker plots show the 25th and 75th percentile range (box) and median values (transverse line in the box). Relative variation was evaluated as: (lytic unit (LU) after intake LU before intake)/LU before intake. Statistical significance of the differences between groups was evaluated by the Mann-Whitney test $(P=0.059)$. $\circ$ Values associated with single individuals.
The relative variations of NK activity in relation to smoking habits after LcS or placebo intake, represented in Fig. 2(b), revealed that the inverse relationship between smoking habits and NK activity was maintained in the placebo group, whereas in the LcS group the NK relative variations did not change in relation to the number of cigarettes smoked. Although the $R$ values of the two regression lines are very similar, the slopes of the two lines are different (regression coefficient $b=-0.071$ for placebo and $b=-0.012$ for LCS). These results indicate that LcS intake increased the relative variations of NK cell activity in smokers.

In order to compare the LcS effect on NK activity in individuals with different smoking habits, it was necessary to compensate the individual differences in smoking habits, and thereby the effect of smoke. Thus, the NK activity of each subject was adjusted, using the regression line, to the value that would have been obtained assuming that the subject did not smoke at all. Fig. 3 illustrates the relative variations of NK activity in the LcS and placebo intake groups adjusted for
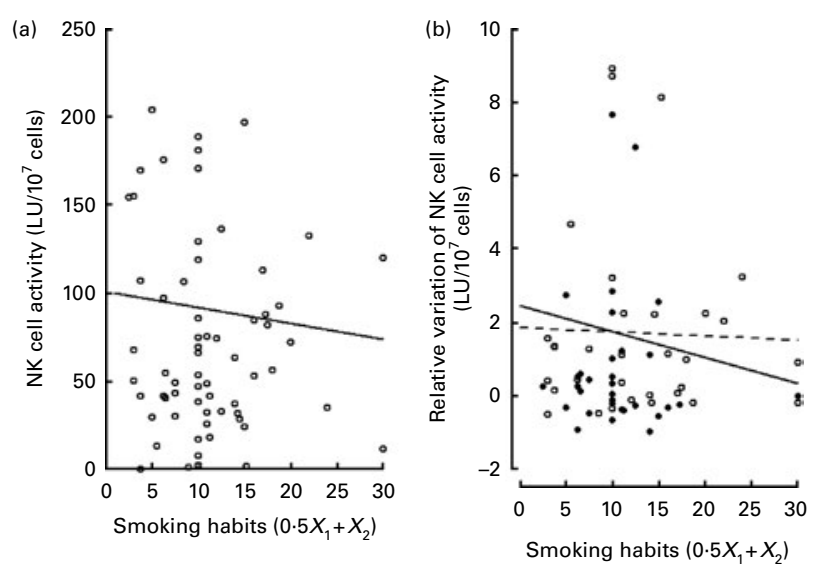

Fig. 2. Relationship between smoking habits and natural killer (NK) cell activity. To represent the long-term smoking habits of each individual, smoking habits were quantified on the basis of number of cigarettes daily smoked $\left(X_{1}\right)$ and the number of smoked cigarettes until giving blood $\left(X_{2}\right)$. (a) NK cell activity before intake in all subjects. The solid line is the estimated regression line, $R-0.064$. (b) Relative variation of NK cell activity in the Lactobacillus casei Shirota (LCS) group (dotted regression line, $R-0.032$, regression coefficient $b=-0.012$ ) and the placebo group (solid regression line, $R-0.028$, regression coefficient $b=-0.071)$. $\circ$, Values associated with single individuals. 
the smoke-related effects. Indeed, negative variations were frequent in the placebo group, while positive variations were observed more frequently in the LcS group. The resulting median values of distribution of differences were -4.38 for the placebo group and 20.98 for the LcS group. The difference between these medians was statistically significant $(P=0 \cdot 039)$.

\section{Effect of Lactobacillus casei Shirota on perception of anxiety, job stress and physical symptoms}

Table 4 shows the results of the questionnaire administered to all enrolled subjects, before and after LcS or placebo intake, to determine their perception of anxiety (STAI I and STAI II), job stress (JD, DL, SS, job insecurity) and subjective perception of physical symptoms. The examined blue-collar workers showed anxiety and occupational stress within the normal range. No significant differences for these parameters were demonstrated after LcS or placebo intake. Interestingly, JD and JS directly correlated with $\mathrm{CD} 16^{+}$cell number (JD, $\rho=0.358, P<0.05$ ) (JS, $\rho=0.389, P<0.05$ ), while an inverse correlation was found between SS and $\mathrm{CD} 16^{+}$cell number only after LcS intake (SS, $\rho=-0.395, P<0.05$ ). Indeed, NK activity is normally reduced in stressful conditions; however, LCS intake may counteract NK activity reduction by mobilisation of an increased $\mathrm{CD} 16^{+}$cell number.

The simultaneous perception of one or more symptoms decreased after both LcS and placebo intake. Since this is a subjective assessment, it is not surprising that there were no obvious differences between the two groups of intake. However, it is to be noted that the percentage of individuals not reporting any specific symptom increased from 11 to $16 \%$ after LCS intake, while this percentage did not change

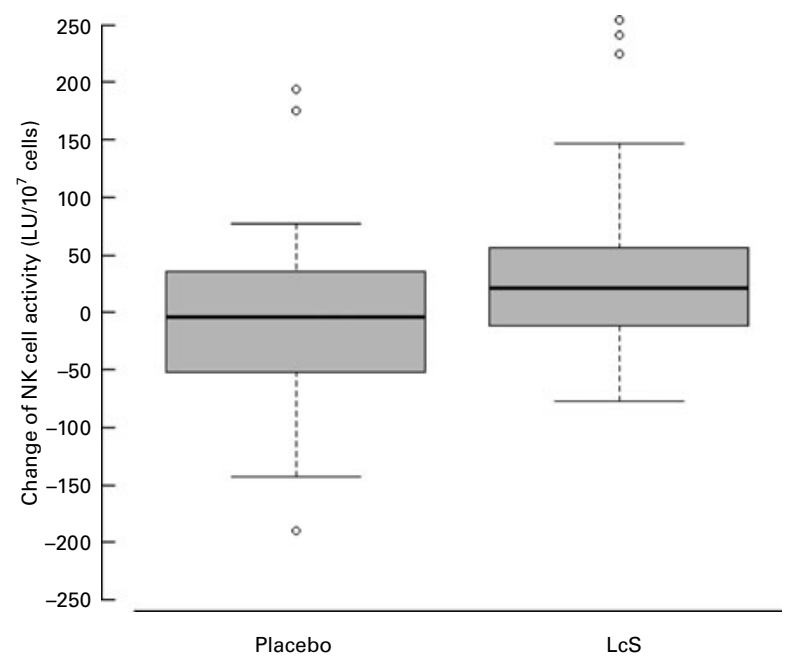

Fig. 3. Changes in natural killer (NK) cell activity before and after intake adjusted for smoking habits. Variations in NK cell activity, measured before and after intake, were adjusted for each individual using the regression line to eliminate the effect of the different smoking habits. Box-whisker plots show the 25th and 75 th percentile range (box) and median values (transverse line in the box, -4.38 and 20.98 for placebo and Lactobacillus case Shirota (LCS), respectively). The difference, calculated by the Mann-Whitney test, between the median values of the two groups was significant, $P=0.039$. $\circ$, Values associated with single individuals. in the placebo group. Table 4 illustrates the percentages of individuals referring representative specific symptoms. It is to be noted that perception of 'nausea and stomach-ache' decreased after LcS intake (from 25.7 to $8.9 \%$ ), and not in the placebo group. Therefore, this result suggests a possible role of LcS intake in reducing gastrointestinal discomfort.

\section{Discussion}

This double-blind, randomised, placebo-controlled study evaluated the effect of 3-week intake of a LcS powder preparation on NK cytotoxic activity in seventy-two Italian male bluecollar volunteers with different smoking habits. The physical and demographic characteristics of the enrolled individuals were similar between the LcS and placebo groups and did not change during the study. LcS intake did not influence leucocyte total number or the relative percentages of neutrophils, eosinophils, monocytes and lymphocytes. On the contrary, the LcS intake significantly increased blood CD16 ${ }^{+}$lymphocytes. $\mathrm{CD}^{+} 6^{+}$cell number was paralleled by increased NKmediated cell cytotoxicity. Some studies indicated that LcS improved NK activity without modification of the cytotoxic cell number ${ }^{(12,27,37)}$. In our study, instead, we observed a significant positive correlation between NK activity and $\mathrm{CD} 16^{+}$ cell number. However, since $\mathrm{CD} 6^{+}$cells include both NK cells, and cells, such as monocytes, capable of enhancing cytotoxic activity ${ }^{(13)}$, we cannot in this study discriminate whether NK activity increase was due directly to an enhancement of the cytotoxic activity of the individual NK cells or to their relative increased number.

The analyses of distribution of changes in NK activity induced by LcS or placebo daily intake revealed that the positive variations of NK activity were more frequent in the LcS group, as compared to the placebo group $(P=0.039)$. This is in agreement with the previous study on Japanese smokers (blue-collar workers) $^{(2)}$. Inverse correlations between the smoked cigarettes and NK cells' activity were demonstrated in all individuals. A smoke-related decrease in NK activity was appreciable in the placebo group, but not in the LcS intake group, indicating that LCS restored NK activity in these smokers. It has been reported that the blue-collar workers have an increased JD and JS in comparison to office employees or schools teachers ${ }^{(24,38)}$. However, the blue-collar workers enrolled in our study resulted in normal psychological conditions, in contrast to their relative poor lifestyle standard. Thus, in these individuals, the LcS treatment did not improve the parameters of anxiety and occupational stress. However, a significantly positive correlation was found between JD and JS and the number of $\mathrm{CD}_{1} 6^{+}$cells in the group treated with LcS. In addition, a significant inverse correlation was demonstrated between SS and $\mathrm{CD}_{1} 6^{+}$cell number, independently from blood cytotoxic activity. This is in agreement with the results of previous studies demonstrating that short, acute psychological stress affects the immune responses by mobilising NK cells ${ }^{(39,40)}$.

Furthermore, LcS treatment appeared to reduce the perception of nausea and stomach-ache symptoms, reflecting an improvement of gastrointestinal functions possibly resulting 
Table 4. Parameters of anxiety, occupational stress and perception of symptoms in the placebo and Lactobacillus casei Shirota (LcS) intake groups (Medians and ranges)

\begin{tabular}{|c|c|c|c|c|c|c|c|c|}
\hline & \multicolumn{4}{|c|}{ Placebo group } & \multicolumn{4}{|c|}{ LcS group } \\
\hline & \multicolumn{2}{|c|}{ Before intake } & \multicolumn{2}{|c|}{ After intake } & \multicolumn{2}{|c|}{ Before intake } & \multicolumn{2}{|c|}{ After intake } \\
\hline & Median & Range & Median & Range & Median & Range & Median & Range \\
\hline \multicolumn{9}{|l|}{ Psychological parameters* } \\
\hline STAI I & 34 & $20-55$ & 34.5 & $22-55$ & 35 & $22-51$ & 33.5 & $23-49$ \\
\hline STAI II & 29 & $20-57$ & 30 & $20-54$ & 30 & $21-42$ & $30 \cdot 5$ & $21-44$ \\
\hline JD & 32 & $24-42$ & 32 & $19-38$ & 31 & $23-42$ & 31.5 & $23-43$ \\
\hline DL & 68 & $48-92$ & 68 & 48-92 & 66 & $52-90$ & 67 & $52-90$ \\
\hline JD:DL & 0.50 & $0.3-0.7$ & 0.50 & $0.3-0.7$ & 0.48 & $0.26-0.67$ & 0.47 & $0.30-0.69$ \\
\hline Social support & 24 & $18-35$ & 24 & $19-36$ & 24 & $19-36$ & 24 & $19-36$ \\
\hline Job insecurity & 10 & $6-20$ & 10 & $6-19$ & 10 & $6-19$ & 9 & $6-19$ \\
\hline \multicolumn{9}{|l|}{ Subjective symptoms perception $\dagger$} \\
\hline Nausea and stomach-ache (\%) & \multicolumn{2}{|c|}{$17 \cdot 6$} & \multicolumn{2}{|c|}{$17 \cdot 6$} & \multicolumn{2}{|c|}{$25 \cdot 7$} & \multicolumn{2}{|c|}{8.9} \\
\hline Back pain $(\%)$ & \multicolumn{2}{|c|}{$64 \cdot 7$} & \multicolumn{2}{|c|}{$56 \cdot 9$} & \multicolumn{2}{|c|}{$60 \cdot 0$} & \multicolumn{2}{|c|}{$55 \cdot 9$} \\
\hline Headache (\%) & \multicolumn{2}{|c|}{$50 \cdot 0$} & \multicolumn{2}{|c|}{47.9} & \multicolumn{2}{|c|}{$42 \cdot 8$} & \multicolumn{2}{|c|}{$35 \cdot 3$} \\
\hline Body weakness (\%) & \multicolumn{2}{|c|}{$14 \cdot 7$} & \multicolumn{2}{|c|}{14.5} & \multicolumn{2}{|c|}{$14 \cdot 3$} & \multicolumn{2}{|c|}{$15 \cdot 0$} \\
\hline
\end{tabular}

STAI, state-trait-anxiety inventory; JD, job demand; DL, decision latitude.

${ }^{*}$ Median values obtained in the state-trait-anxiety inventory and Karasek questionnaires.

$\dagger$ Percentages of individuals reporting the specific subjective symptom.

from a restored microbiota balance. Although it is well established that ingested LcS transits alive through the stomach and survives in the intestine ${ }^{(41)}$, it is still unclear as to how LcS may improve the upper gastrointestinal tract symptoms, such as nausea or stomach-ache. Direct interaction of LcS with the stomach or indirect action through hormonal or nervous regulation may be assumed and may represent future research subjects.

In conclusion, this study demonstrated that daily LcS intake significantly increased NK cytotoxic activity and $\mathrm{CD}_{16}{ }^{+}$cell number. Moreover, a reduced symptom perception in healthy Italian habitual smokers was reported. Since smokers exhibit higher susceptibility to infections and inflammatory diseases and NK cells play an important role in controlling infections and eliminating aberrant cells, dietary supplementation with LcS could contribute to increased NK cytotoxic activity with significant health advantages for individuals with smoking habits. However, these results should not be interpreted as an attempt to overcome smoke-related damages, but on the contrary they strengthen the notion of tobacco interference with the immune system. Smoking habit and alcohol consumption are the two most important preventable causes of disease and premature death. Despite increasing knowledge of their hazard, prevalence is declining very slowly and their decrease appears to be limited to the higher socio-economic class among populations. Smoking threats are not limited to the immunosuppressive effect, but have a major health impact. Thus, despite the LcS increase of NK activity, a concerted effort to quit smoking is the only way to real prevention of cancer and CVD.

\section{Acknowledgements}

The authors thank Yakult Honsha Company Limited for providing the powder preparations containing LCS and the placebo. The authors also thank Dr Masanobu Nanno from the Yakult Central Institute for Microbiological Research
(Tokyo, Japan), Dr Kaori Suzuki from Yakult Europe B.V. and Dr Anna Castioni from Yakult Italia for helpful suggestions and logistic support. M. R. coordinated the experimental tests, and contributed to data analyses and manuscript preparation; P. B. conceived the study and recruited the subjects; V. B. and C. T. carried out the laboratory analyses; M. D. N. carried out the statistical analyses; L. F. administered and analysed the physiological tests; Q. L. and K. M. contributed to setting up laboratory protocols; and R. M. coordinated the research, data analyses and manuscript preparation. All authors read and approved the final manuscript. P. B. and R. M. had research funding by Yakult Honsha Company Limited, and K. M. received consultancy fees from Yakult Honsha Company Limited. M. R., V. B., C. T., M. D. N., L. F. and Q. L. have no conflicts of interest to declare.

\section{References}

1. Oelschlaeger TA (2010) Mechanisms of probiotic actions: a review. Int J Med Microbiol. 300, 57-62.

2. Morimoto K, Takeshita T, Nanno M, et al. (2005) Modulation of natural killer cell activity by supplementation of fermented milk containing Lactobacillus casei in habitual smokers. Prev Med 40, 589-594.

3. Rao AV, Bested AC, Beaulne TM, et al. (2009) A randomized, double blind, placebo-controlled pilot study of a probiotic in emotional symptoms of chronic fatigue syndrome. Gut Pathol 19, 1-6.

4. Ng SC, Hart AL, Kamm MA, et al. (2009) Mechanisms of action of probiotics: recent advances. Inflamm Bowel Dis 15, 300-310.

5. Hormannsperger G \& Haller D (2010) Molecular cross-talk of probiotic bacteria with the intestinal immune system: clinical relevance in the context of inflammatory bowel disease. Int J Med Microbiol 300, 63-73.

6. So JS, Lee CG, Kwon HK, et al. (2008) Lactobacillus casei potentiates induction of oral tolerance in experimental arthritis. Mol Immunol 46, 172-180. 
7. Mohamadzadeh M, Olson S, Kalina WV, et al. (2005) Lactobacilli activate human dendritic cells that skew $\mathrm{T}$ cells toward T helper1 polarization. Proc Natl Acad Sci U S A 102, 2880-2885.

8. Ghadimi D, Folster-Holst R, de Vrese M, et al. (2008) Effect of probiotic bacteria and their genomic DNA on Th1/Th2-cytokine production and peripheral blood mononuclear cells (PBMCs) on healthy and allergic subjects. Immunobiology 21, 677-692.

9. Kwon HK, Lee CG, So JS, et al. (2010) Generation of regulatory dendritic cells and $\mathrm{CD}^{+} \mathrm{Foxp}^{+} \mathrm{T}$ cells by probiotic administration suppresses immune disorders. Proc Natl Acad Sci U S A 107, 2159-2164.

10. Cross ML, Stevenson LM \& Gill HS (2001) Anti-allergy properties of fermented foods: an important immunoregulatory mechanism of lactic acid bacteria? Int Immunopharmacol 1, 891-901.

11. Maasen CB, van Holten-Neelen C, Blak F, et al. (2000) Straindependent induction of cytokine profiles in the gut by orally administrated Lactobacillus trains. Vaccine 18, 2613-2623.

12. Takeda K, Suzuki T, Shimada K, et al. (2006) Interleukin-12 is involved in the enhancement of human natural killer cell activity by Lactobacillus casei Shirota. Clin Exp Immunol 146, 109-115.

13. Dong H, Rowland I, Tuohy KM, et al. (2010) Selective effects of Lactobacillus casei Shirota on T cell activation, natural killer cell activity and cytokine production. Clin Exp Immunol 16, 378-388.

14. Sheih YH, Chiang BL, Wang LH, et al. (2001) Systemic immunity-enhancing effects in healthy subjects following dietary consumption of the lactic acid bacterium Lactobacillus rhamnosus HNO01. J Am Coll Nutr 20, 149-156.

15. Gill HS, Rutherfurd KJ \& Cross ML (2001) Dietary probiotic supplementation enhances natural killer cell activity in the elderly: an investigation of age-related immunological changes. J Clin Immunol 21, 264-271.

16. Ogata K, An E, Shioi Y, et al. (2001) Association between natural killer cell activity and in infection in immunologically normal elderly people. Clin Exp Immunol 124, 392-397.

17. Nova E, Wanberg J, Gomez-Martinez S, et al. (2007) Immunomodulatory effects of probiotics in different stages of life. Br J Nutr 98, 90-95.

18. Miller GE, Chen E, Fok AK, et al. (2009) Low early-life social class leaves a biological residue manifested by decreased glucocorticoid and increased proinflammatory signalling. Proc Natl Acad Sci U S A 106, 14716-14721.

19. Schleifer SJ, Keller SE, Bartlett JA, et al. (1996) Immunity in young adults with major depressive disorder. $\mathrm{Am}$ J Psychiatry 153, 477-482.

20. Inoue-Sakurai C, Maruyama S \& Morimoto K (2000) Posttraumatic stress and lifestyles are associated with natural killer cell activity in victims of the Hanshin-Awaji earthquake in Japan. Prev Med 31, 467-473.

21. Witek-Janusek L, Gabram S \& Mathews HL (2007) Psychologic stress, reduced NK cell activity and cytokine disregulation in women experiencing diagnostic breast biopsy. Psychoneuroendocrinology 32, 22-35.

22. Morikawa Y, Kitaoka-Higashiguchi K, Tanimoto $\mathrm{C}$, et al. (2005) A cross-sectional study on the relationship of job stress with natural killer cell activity and natural killer cell subsets among healthy nurses. J Occup Health 47, 378-383.

23. Okamoto H, Tsunoda T, Teruya K, et al. (2008) An occupational health study of emergency physicians in Japan: health assessment by immune variables (CD4, CD8, CD56, and NK cell activity) at the beginning of work. $J$ Occup Health 50, 136-146.
24. Boscolo P, Di Donato A, Di Giampaolo L, et al. (2009) Blood natural killer activity is reduced in men with occupational stress and job insecurity working in a university. Int Arch Occup Environ Health 82, 787-794.

25. Morimoto K, Takeshita T, Inoue-Sakurai C, et al. (2001) Lifestyle and mental health status are associated with natural killer cell and lymphokine activated killer cell activities. Sci Total Environ 270, 3-11.

26. Li Q, Morimoto K, Nakadai A, et al. (2007) Healthy lifestyles are associated with higher levels of perforin, granulysin and granzymes A/B-expressing cells in peripheral blood lymphocytes. Prev Med 44, 117-123.

27. Nagao F, Nakayama M, Muto T, et al. (2000) Effects of fermented milk drink containing Lactobacillus casei strain Shirota on the immune system in healthy human subjects. Biosci Biotechnol Biochem 64, 2706-2798.

28. Li Q, Morimoto K, Nakadai A, et al. (2007) Forest bathing enhances human natural killer activity and expression of anti-cancer proteins. Int J Immunopatbol Pharmacol 20, 3-8.

29. Li Q, Morimoto K, Kobayashi M, et al. (2008) Visiting a forest, but not a city, increases human natural killer activity and expression of anti-cancer proteins. Int J Immunopathol Pharmacol 21, 117-128.

30. Firoz Mian M, Lauzon NM, Stampfli MR, et al. (2008) Impairment of human NK cell cytotoxic activity and cytokine release by cigarette smoke. J Leukoc Biol 83, 774-784.

31. Lu LM, Caleb CJ, Zavitz CJ, et al. (2007) Cigarette smoke impairs NK cell-dependent tumor immune surveillance. J Immunol 178, 936-943.

32. Spielberger CD, Gorsuch RL \& Lushene RE (1970) Manual of the State-Trait-Anxiety-Inventory. Palo Alto, CA: Consulting Psychologists Press.

33. Spielberger CD (1989) Inventario per l'Ansia di Stato e di Tratto (Inventory for State and Trait Anxiety). Firenze: O.S. Organizzazioni Speciali.

34. Derogatis LR (1983) SCL-9O Administration, Scoring and Procedures. Manual II. Towson, MD: Clinical Psychometric Research.

35. Karasek RA, Brisson C, Kawakami N, et al. (1998) The Job Content Questionnaire (JCQ): an instrument for internationally comparative assessments for internationally comparative assessments of psychosocial job characteristics. I Oсcup Health Psychol 3, 322-355.

36. Baldasseroni A, Camerino D, Cenni P, et al. (2001) La valutazione dei fattori psicosociali - Il Job Content Questionnaire (The evaluation of psychosocial factors - The Job Content Questionnaire). Fogli d'Informazione 3, 20-32.

37. Matsuzaki T, Saito M, Usuku K, et al. (2005) A prospective uncontrolled trial of fermented milk drink containing viable Lactobacillus casei stain Shirota in the treatment of HTLV-1 associated myelopathy/tropical spastic paraparesis. J Neurol Sci 237, 75-81.

38. Forcella L, Di Donato A, Reversi S, et al. (2009) Occupational stress, job insecurity and perception of the health status in Italian teachers with stable or temporary employment. J Biol Regul Homeost Agents 23, 85-93.

39. Bosch JA, Berntson GG, Cacioppo JT, et al. (2005) Differential mobilization of functionally distinct natural killer subsets during acute psychologic stress. Psychosom Med 67, 366-375.

40. Boscolo P, Di Gioacchino M, Reale R, et al. (2011) Work stress and innate immune response. Int J Immunopath Pharmacol 24, 51-54.

41. Yuki N, Watanabe K, Mike A, et al. (1999) Survival of a probiotic, Lactobacillus casei strain Shirota, in the gastrointestinal tract: selective isolation from faeces and identification using monoclonal antibodies. Int J Food Microbiol 48, 51-57. 